\title{
La planificación estratégica como herramienta de desarrollo de las empresas agropecuarias en el Ecuador
}

\author{
Strategic planning as a development tool for agricultural companies in \\ Ecuador
}

Edison Ruperto Carrillo Parra. ${ }^{1}$, Ángel Eduardo Rodríguez Solarte. ${ }^{2}$ \& Mario Alfonso Arellano Díaz. ${ }^{3}$

\begin{abstract}
.
DOI: $\underline{\text { https://doi.org/10.33262/visionariodigital.v4i3.1361 }}$

This work is the summarized result of the degree work called "Strategic plan to improve the efficiency of financial administrative processes in the dairy company" CAMPO FINO ", of the city of Salcedo during the period 2018 - 2023" carried out by Eng Ing. Ligio Elena Mora Cevallos, as part of her degree work, under the guidance of the undersigned, and seeks to develop tools that strengthen the financial administrative processes of the dairy company "CAMPO FINO", since this business organization does not have a strategic plan that allows to enhance its processes and therefore improve the results of its management.

The first phase was the application of a diagnosis that determines the administrative and financial status. The second phase is the development of a proposal aimed at solving the problems found in the diagnosis.

For the development of this work, a participatory methodology will be used whose fundamental actors are the collaborators of the operational and administrative area of the company, as well as the criteria of its clients, regarding what is the expectation regarding the level of service, and what they expect from the products that the company has placed in the markets.
\end{abstract}

1 Escuela Superior Politécnica de Chimborazo, Facultad de Ciencias Pecuarias, Riobamba, Ecuador edison.carrillop@espoch.edu.ec

${ }^{2}$ Escuela Superior Politécnica de Chimborazo, Facultad de Administración de Empresas, Riobamba, Ecuador, rodriguez@espoch.edu.ec

${ }^{3}$ Escuela Superior Politécnica de Chimborazo, Facultad de Administración de Empresas, Riobamba, Ecuador, mario.arellano@espoch.edu.ec 
Finally, to prepare the proposal we will start from the different concepts and models established, accepted and applied by successful companies, adapted to the reality of our environment and the idiosyncrasy of our people, factors that are fundamental in the design of efficient strategies that allow attracting the largest number of customers, in the local, regional, national environment, this will undoubtedly underpin the corporate image of the company, which in turn will allow the development of a brand with identity and positioned in the market.

Keywords: Strategic planning, strategic diagnosis, administrative process, Control systems.

\section{Resumen.}

El presente trabajado es el resultado resumido del Trabajo de titulación denominado "Plan estratégico para mejorar la eficiencia de los procesos administrativos financieros en la empresa de lácteos "CAMPO FINO”, de la ciudad de Salcedo durante el periodo 2018 - 2023” realizado por la Ing. Ing. Ligio Elena Mora Cevallos, como parte del su trabajo de titulación, bajo la tutoría del suscrito, y busca desarrollar herramientas que fortalezcan los procesos administrativos financieras de la empresa de lácteos "CAMPO FINO”, puesto que esta organización empresarial no cuenta con un plan estratégico que permita potencializar sus procesos y por ende mejorar los resultados de su gestión.

La primera fase fue la aplicación de un diagnóstico que nos determine cuál es el estado administrativo y financiero. La segunda fase es la elaboración de una propuesta orientada a resolver los problemas encontrados en el diagnóstico.

Para el desarrollo de este trabajo se utilizará una metodología participativa cuyos actores fundamentales son los colaboradores del área operativa y administrativa de la empresa, así como también el criterio de sus clientes, con respecto a cuál es la expectativa en lo que se refiere al nivel de servicio, y que es lo que esperan de los productos que la empresa tiene colocados en los mercados.

Finalmente, para la elaboración de la propuesta partiremos de los diferentes conceptos y modelos establecidos, aceptados y aplicados por empresas exitosas, adaptados a la realidad de nuestro medio y a la idiosincrasia de nuestra gente, factores que son fundamentales en el diseño de estrategias eficientes que permitan captar la mayor cantidad de clientes, en el entorno local regional, nacional, esto sin duda apuntalara la imagen corporativa de la empresa lo que a su vez permitirá el desarrollo de una marca con identidad y posicionada en el mercado.

Palabras claves: Planificación estratégica, diagnóstico estratégico, proceso administrativo, Sistemas de control. 


\section{Introducción.}

La producción e industrialización lacte en el ecuador es una de las principales generadoras de fuente de trabajo en el país, según el Banco Central en su estudio realizado en el año 2015 sobre la cadena productiva de la leche determinó que genera el rededor de 1,5 millones de fuentes directas e indirectas de trabajo y aporta al 8\% del PIB.,

En este sentido según la revista EKOS (2019) la producción de leche por regiones está compuesta de la siguiente manera: la sierra produce el $73 \%$ de leche, en la Costa el 19\% y en la Amazonía 8\%. Y del total nacional el 70\% de la producción está concentrado en los pequeños productores. Esta información nos permite analizar que es urgente redefinir la estrategia productiva y fomentar el incremento de la productividad a través de la formulación de políticas públicas que apoyen a este sector vulnerable de la economía nacional.

Otro aspecto importante a analizar es el sistema de industrialización de la leche, según un estudio de la FAO (2018), en el ecuador se producen alrededor de 4.982.370,00 litros diarios, de los cuales 2,662.560 litros son procesados, de acuerdo al siguiente detalle: $31 \%$ corresponde a Queso, $27 \%$ a leche en funda, $20 \%$ a leche en cartón, el $11 \%$ en leche en polvo, el $10 \%$ a yogurt y el $1 \%$ a otros productos, gran parte de los sub productos lácteos que se comercializan, proviene de pequeñas empresas rurales y/o familiares cuyos procesos administrativos aun no son óptimos lo que afecta directamente a sus sostenibilidad económica y financiera.

En este contexto resulta urgente implementar sistemas administrativos eficientes que mejoren significativamente sus procesos operativos administrativos, cuyo efecto tiene que observarse en los resultados económicos financieros de las empresas, razón por la que el presente trabajo investigativo se concentra en la empresa "CAMPO FINO" ubicada en la provincia de Cotopaxi, cantón Salcedo, cuya actividad económica se inicia en agosto del 2005, produciendo Queso Yogur, leche fresca en fundas y refrescos, en la actualidad sigue la misma línea de producción con excepción de los refrescos.

\section{Objetivos.}

\section{General.}

Fortalecer los procesos administrativos financieros, a través del diseño de herramientas administrativas, que garantice la sostenibilidad financiera de la empresa procesadora de láctea "Campo Fino".

\section{Específicos.}

- Realizar un diagnóstico a la empresa Campo Fino que permita determinar la realidad Administrativa y operativa y su efecto sobre los resultados económicos financieros de la empresa. 
- Diseñar un plan estratégico que determine el rumbo a seguir partiendo del análisis de sus fortalezas y debilidades.

\section{Fundamento teórico.}

\section{Planificación.}

Graffe (2006), citado en Mora (2019) afirma que la planificación es la actividad humana de carácter racional que tiende a la definición de una conducta a seguir con el objeto de lograr la transformación de la realidad, para lo cual se definen y coordinan objetivos a lograr, sobre la base del análisis previo de los hechos observados en la realidad presente y sus condicionamientos históricos y de la proyección de su tendencia que observa el sistema a planificar. (pp. 2-3)

(Scanlan, K; citado en Mora 2019) afirma: "sistema que comienza con los objetivos, desarrolla políticas, planes, procedimientos y cuenta con un método de retroalimentación de información para adaptarse a cualquier cambio en las circunstancias" (p. 5).

(Mercado, S; citado en Mora 2019) Planear es definir los objetivos y determinar los medios para alcanzarlos; es fundamentalmente analizar por anticipado los problemas, planear posibles soluciones y señalar los pasos necesarios para llegar con eficiencia a los objetivos que la solución elegida define. La planeación debe, por tanto, fijar el curso concreto de acción que ha de seguirse, estableciendo los principios que habrán de orientarlo, la secuencia de operaciones para realizarlo y la determinación del tiempo necesario para su planeación. (p.5).

\section{Clasificación de la planificación.}

Según Graffe (2006) la planificación se clasifica en:

Planificación Normativa. Se define como un proceso metódico que permite definir objetivos y señala el camino como alcanzarlos, minimizando costos y maximizando beneficios.

Planificación situacional. Es un proceso continuo y sistemático, que permite que, a través del análisis y la discusión se determine la dirección que guíe el cambio situacional de una empresa.

Planificación corporativa. Esta planificación propia de las grandes empresas busca disminuir el riesgo de las amenazas y aprovechar las fortalezas que le brinda su entorno. (pp. 15-16)

\section{Definición de planificación estratégica.}

Armijo (2014), citado en Mora (2019) manifiesta que la PE es una herramienta de gestión que apoya la toma de decisiones en las organizaciones y se basa en la formulación de objetivos de carácter prioritario, apoyados por estrategias que definen con claridad cuál es el camino para recorrer para alcanzar los objetivos propuestos. 


\section{Fases o dimensiones de la panificación estratégica.}

Sierra (2008), citado en Mora (2019) indica que existen diferentes aspectos a considerar en la formulación de un plan estratégico los mismos que se detallan a continuación:

- Mora (2019) manifiesta que Fase filosófica es el diseño o ajuste de la misión, visión, valores y políticas institucionales o corporativas.

- Fase Analítica: integrada por la definición de objetivos organizacionales, el análisis del entorno externo e interno de la organización en su situación presente, prospectiva, y la determinación de estrategias para la consecución de los objetivos establecidos.

- Fase Táctica u Operativa: Comprende la definición del plan de acción (actividades y responsables), asignación presupuestaria de inversión para la implementación del respectivo plan estratégico. Este proceso se puede observar en la siguiente figura. (pp. 66)

Figura 1. Proceso de planeación estratégica

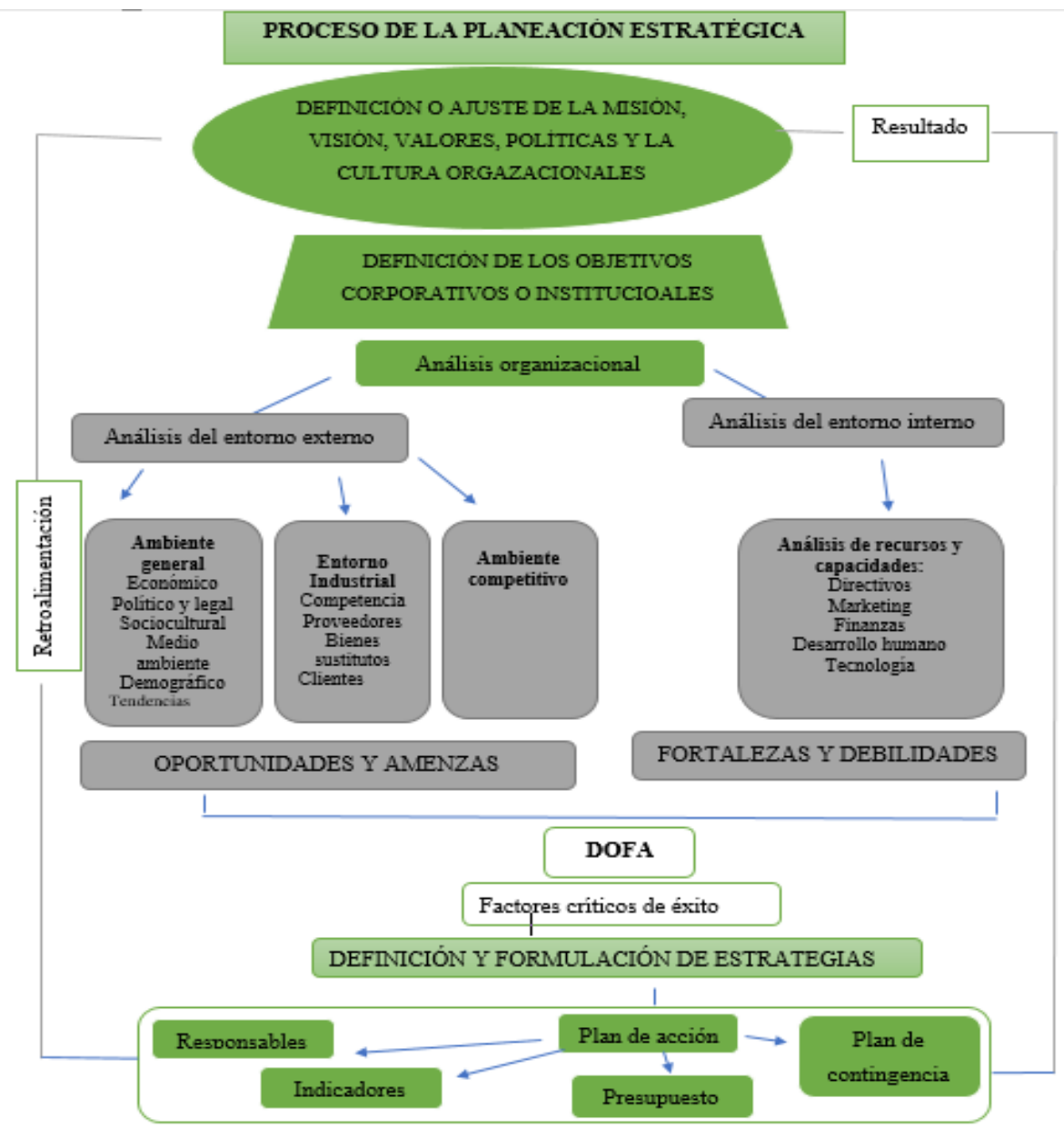

Fuente: Proceso administrativo para las organizaciones del siglo XXI. Sierra \& Bernal 2008 


\section{Fase filosófica.}

Sierra (2008), citado en Mora (2019) define a esta fase como la declaraciòn de la filosofia de la organizaciòn, en donde todos los aspectos de la organizaciòn deben estar en cocncordancia y alineados con la folosofia de la organizaciòn, y esta integrado por:

- Misión

- Visión

- Valores y la cultura organizacional

- Políticas. (p. 68)

Figura 2. Aspectos de la fase filosófica de la planeación estratégica

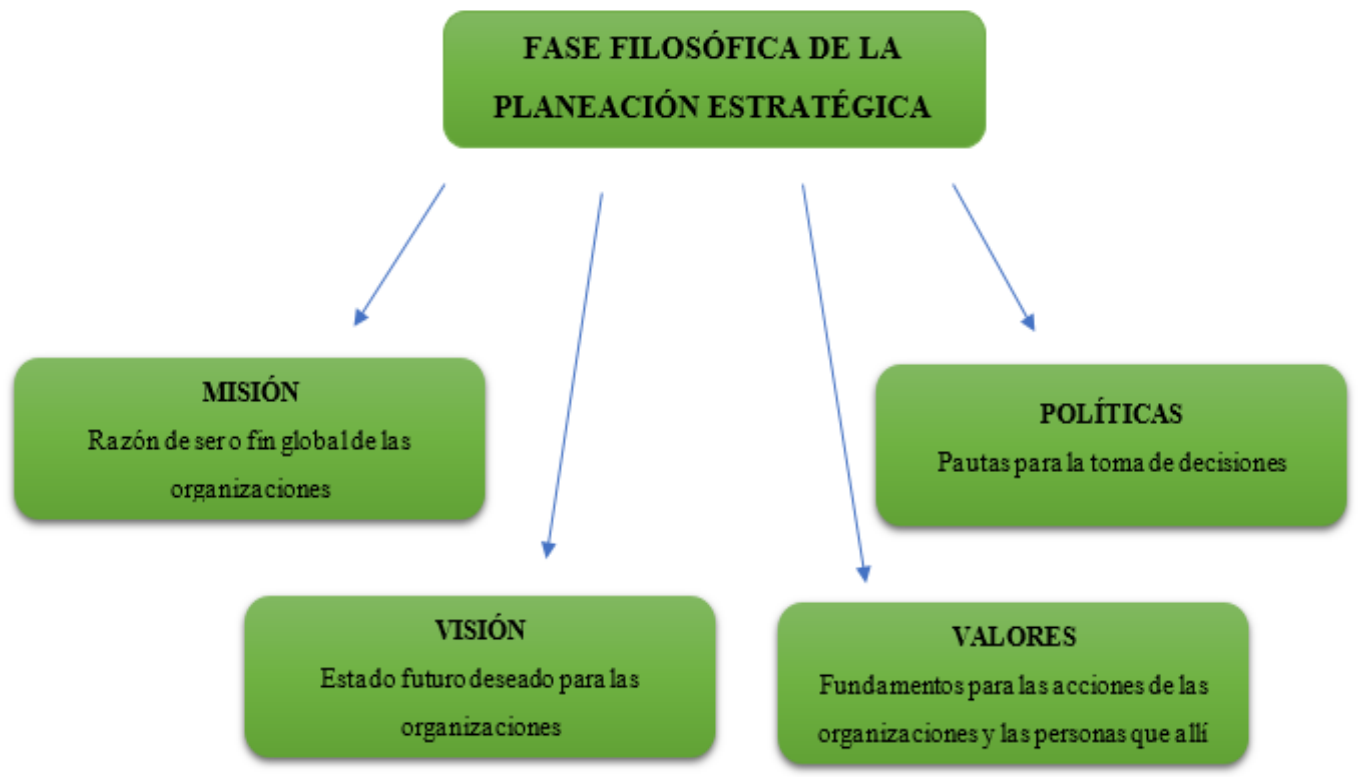

Fuente: Proceso administrativo para las organizaciones del siglo XXI. Sierra \& Bernal 2008

\section{Fase analítica.}

Sierra (2008) manifiesta que en esta fase se desarrolla los puntos que a continuación se describen:

- Definición de objetivos institucionales denominados también corporativos, los mismos que buscan dar solución al problema encontrados en el diagnostico.

- Elaboración del análisis interno y externo de la organización y su entorno.

- Definición e implementación de estrategias que permitan alcanzar los objetivos. 
Figura 3. Fase analítica de la planeación estratégica

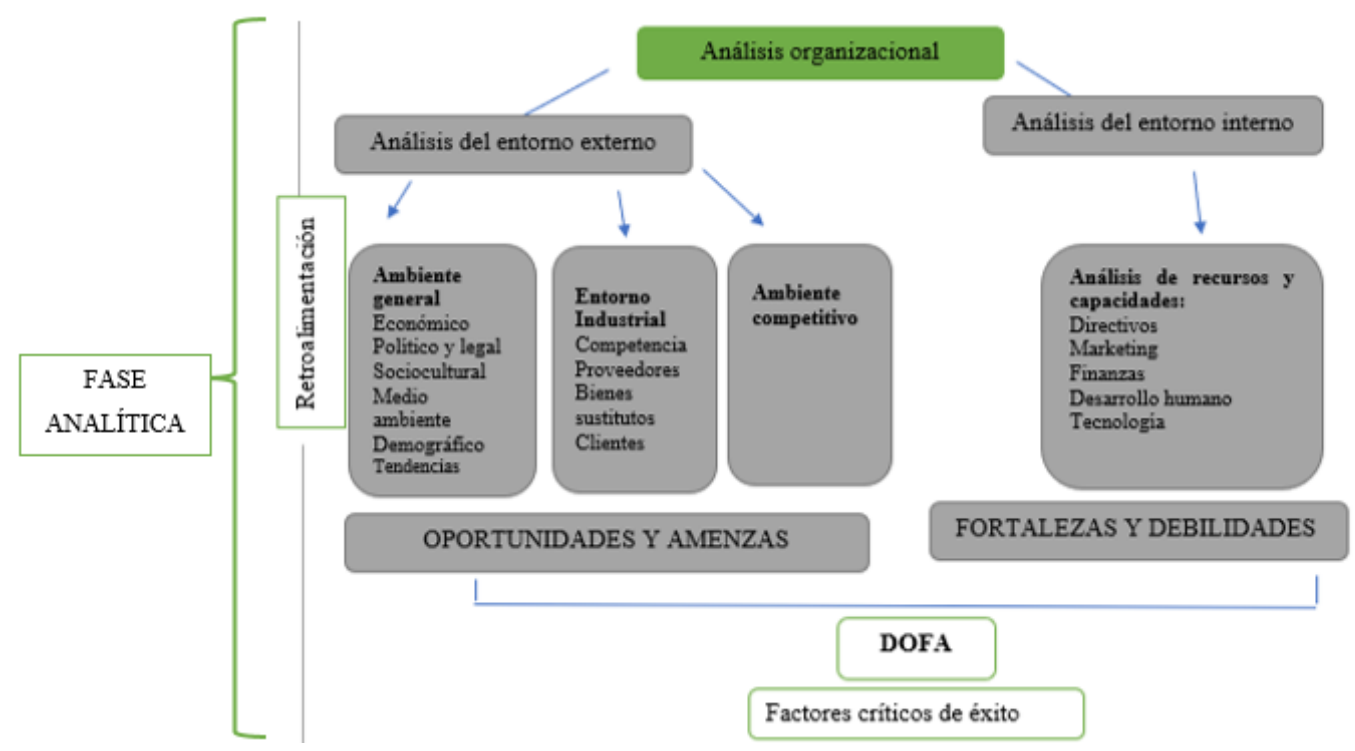

Fuente: Proceso administrativo para las organizaciones del siglo XXI. Sierra \& Bernal 2008

\section{Fase operativa.}

Sierra (2008) señala que en esta fase se define las actividades que permitan alcanzar los objetivos, pero es indispensable considerar que las actividades, deben estar alineadas estratégicamente hacia los objetivos, evitando que existan acciones que no sumen $\mathrm{y}$, al contrario, generen gastos innecesarios. Es importante también aclarar que todas las actividades definidas deben tener asignado un presupuesto que garantice su ejecución.

\section{Planeación estratégica de Mintzberg como lo citó Chiavenato.}

Chiavenato (2011), define que el modelo de la planeaciòn estrategica considera las siguientes etapas:

- Declaración de la misión: En esta etapa se define la responsabilidades de la organización en su entorno, la misión nos permite de manera organizada a la empresa orientar las actividades.

- Visión de los negocios: Nos indica gacia donde quiere llegar la organizaciòn en un periodo de tiempo especifico

- Diagnóstico estratégico externo: Define el efecto que tienne las variables externas sobre el funcionamiento de la organizaciòn, se le denomina tambien conoi analisis del micro entorno, y se analiza las amanezas y las oportunidades, este ejercicio es importante porque nos permite reorientar la misiòn y visiòn. 
- Diagnóstico estratégico interno: se refiere al analisìs de los fortalezas y debilidades que tiene la organizaciòn, el resultado nos permite definir las estrategias mas eficientes que permitan confrontar los problemas y potenbcuiliazr las fortalezas de la empresa.

- Determinantes del éxito: Para Mora (2019), Se refiere a la inclusiòn de la evaluación, la misma que se ubicada entre el diagnóstico, definición de objetivos y formulación de estrategias. También son llamadas factores críticos del éxito y son la base de las políticas de los negocios.

- Definición de los objetivos: esta definiciòn nos permite alxanzar la visiòn de la organizaciòn, estos deben ser alcanzados en un perior especifico de tiempo.

- Formulación de estrategias: La formulación estratégica nos señala el camino que debemos seguir para alcanzar los objetivos

- Desempeño Estratégico: Para Mora (2019), El plan estratégico es un plan de acción, no basta con formular las estrategias, sino que se deben implementar por medio de programas y proyectos.

- Auditoría del Desempeño y resultados: Es el control de la ejecuciòn de las estrategias, y el comportamiento que estas tiene con respectoo a alcanzar los resultado planteados como parte de la implementaciòn de la PE. (pp. 46-47)

Figura 4. Modelo de Planeación Estratégica de Mintzberg

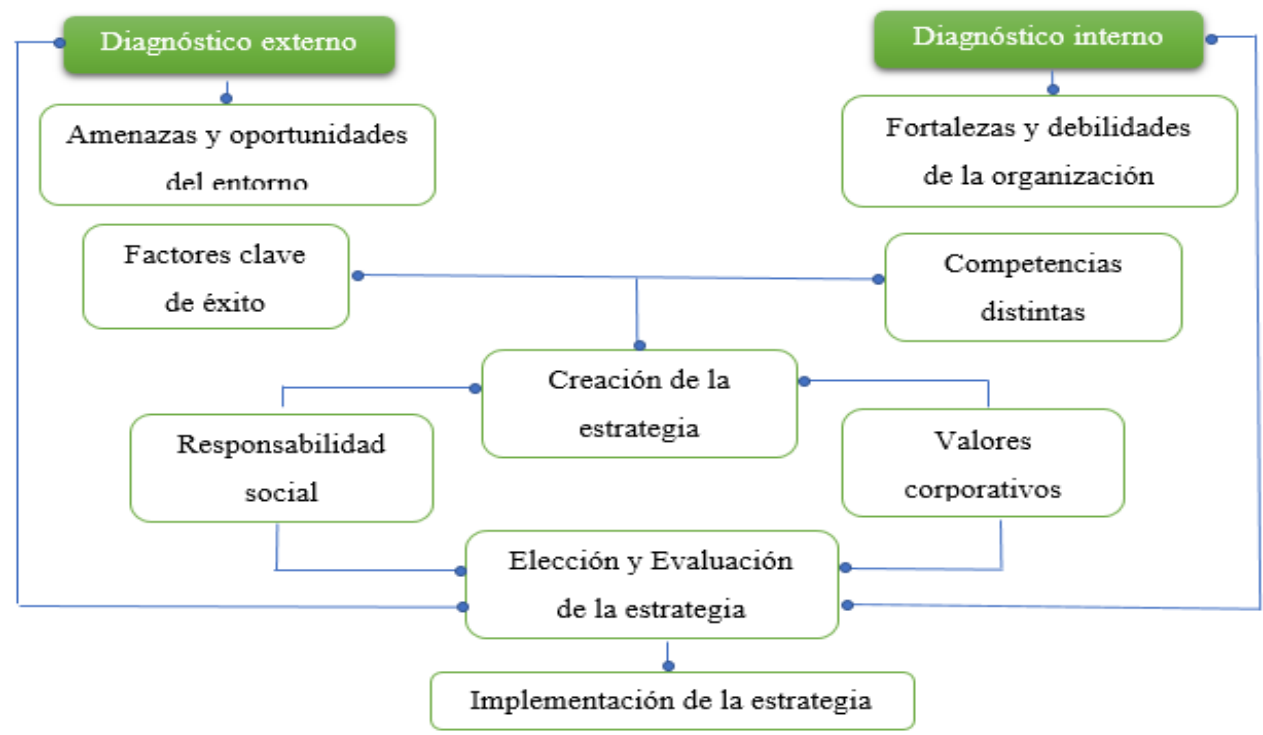

Fuente: Planeación Estratégica fundamentos y aplicaciones. Chiavenato Idalberto 2011 


\section{Metodología.}

\section{Modalidad de la investigación.}

\section{Enfoque cualitativo.}

Este tipo de investigación se guía por áreas de estudios cuya particularidad permite generar preguntas e hipótesis antes, durante y después de la recolección de datos, su principal ventaja radica en que, a través de preguntas acertadas permite analizar los hechos y los procesos; utiliza como herramienta la observación.

\section{Tipos de investigación.}

Se utilizará la investigación exploratoria, descriptiva, y explicativa, la primera investiga aspectos de la empresa que aún no han sido analizados permitiendo explorar aspectos generales de la empresa y deja el canal abierto para que investigaciones posteriores realicen estudios de aspectos específicos.

La segunda hacer referencia a una descripción concreta de los hechos sin que esto signifique analizar las causas del problema, la tercera intenta explicar las causas de los problemas y sus posibles soluciones. A partir de estos criterios se tratará de encontrar las principales dificultades por las que atraviesa le empresa.

\section{Diseño de la investigación.}

\section{Investigación no experimental.}

(Sampieri 2014, citado en Mora 2019) define a esta investigación, como el proceso que no manipula deliberadamente variables. Es decir, son estudios en los que no se manipula en forma intencional las variables independientes para ver su efecto sobre otras variables.

Lo que hacemos en la investigación no experimental es observar fenómenos tal como se dan en su contexto natural, para analizarlos. Partiendo de este concepto el presente estudio partirá de la realidad existente lo que permitirá explorar los problemas en sus reales dimensiones y a partir de este análisis construir propuestas de mejora a los diferentes procesos administrativos financieros de la empresa.

\section{Población y muestra.}

La empresa cuenta con 20 colaboradores más 2 directivos y los dueños, dada la cantidad de empleados y considerando el criterio participativo todos los que conforma la empresa serán parte de la construcción del diagnóstico y del plan estratégico.

\section{Métodos técnicas e instrumentos.}




\section{Métodos.}

Se recurrirá al método inductivo porque utiliza el razonamiento a partir de hechos aceptados como válidos para la construcción de conclusiones, en nuestro caso permitirá analizar proceso individual para determinar sus debilidades, fortalezas y como estos afectan ya sea positiva o negativamente en la gestión de la empresa campo fino.

\section{Técnicas.}

Guerrero (2016) presenta las siguientes técnicas:

- Observación. Permite captar mediante la vista fenómenos y hechos en la cotidianidad de la naturaleza o sociedad.

- Entrevista. Utiliza como herramienta el diálogo sobre temas específicos que permite auscultar la realidad de un hecho, este técnico está orientada especialmente a los directivos y dueños de la empresa.

- Encuesta. Esta herramienta se basa en una serie de preguntas previamente establecidas, y orientadas a la obtención de información clave sobre el funcionamiento de la empresa este técnico estará dirigida a los colaboradores que realizan el trabajo operativo en la empresa.

\section{Resultados y discusión.}

\section{Plan estratégico.}

\section{Análisis situacional.}

\section{Análisis del entorno empresarial.}

Este modelo a su vez se subdivide en dos elementos de análisis, el macro ambiente y el microambiente Su importancia radica en que permite conocer el entorno y cómo afecta al sector lácteo. También, facilita el conocimiento del nivel de competitividad de la empresa, así como sus fortalezas y debilidades.

\section{Macro ambiente.}

El macro ambiente analiza el conjunto de fuerzas de carácter económico, político, social, cultural, demográfico, jurídico y tecnológico, que afectan en forma individual o en interacciona a la empresa, así por ejemplo lo económico está íntimamente ligado a lo político y estos dos factores afectan directa mente a lo social. Por esta razón muchas empresas realizan un seguimiento al entorno por el impacto que tienen sobre su sostenibilidad, es decir permiten determinar las amenazas y oportunidades que genera el macroentorno sobre su empresa. 
Factor demográfico.

Según información publicada por el Instituto de Estadísticas y Censos (INEC) la población estimada para el 2020 es de 17.510.643,00 habitantes, al comparar la población del año 2019 se observa un crecimiento poblacional del $1,56 \%$ esto significa que en 12 meses se incrementaron 242.657,00 habitantes. Este crecimiento sostenido de la población tanto a nivel rural como urbano en nuestro país tiene un impacto directo sobre la demanda de productos alimenticios.

\section{Factor Económico.}

La primera variable analizada es el producto interno bruto, Según datos del Banco el país registrar un decrecimiento en su producto interno bruto (PIB) especialmente por efectos de la pandemia y por la profunda crisis económica por la que atraviesa antes y después de la pandemia, el país, en base a este análisis las proyecciones de crecimiento económico del Ecuador para el cierre de 2020 son negativas.

Según el Banco Central del Ecuador (BCE), el producto interno bruto (PIB) tendrá un crecimiento negativo del 7,0 \%, mientras que el Banco Mundial (BM) y el Fondo Monetario Internacional (FMI) estiman un decrecimiento del 6,9\% y 6.30\% respectivamente, esto sin duda ensombrece mucho el panorama económico para el país. En cuanto al empleo según las últimas cifras del Instituto Nacional de Estadística y Censos, el empleo adecuado disminuyó al pasar de 40,6\% en diciembre 2018 a 38,8\% en el mismo mes de 2019.

Esta es la tasa más baja registrada en un cierre de año desde 2007 y se explica por la situación que enfrenta el mercado laboral en Ecuador.

Por otro lado, el empleo no adecuado incrementó al pasar de 55,3\% a 56,7\% entre diciembre 2018 y diciembre 2019. Dentro del empleo no adecuado, la categoría subempleo por insuficiencia de trabajo es la que presenta mayor aumento que las otras categorías, pasando de 13,4\% a 14,7\% entre diciembre de 2018 y 2019. La tasa de desempleo cerró diciembre de 2019 en 3,8\%, es decir 0,1 puntos porcentuales con relación al mismo periodo de 2018.

\section{Factor político.}

Tiene un efecto negativo sobre nuestra economía, considerando que estamos frente a un gobierno cuyas política económica ha favorecido los interés de: la Banca, grandes empresarios, industriales, importadores y exportadores, generando una crisis económica a los sectores más vulnerables del país, sumado a eso los actos de corrupción denunciados, esto sin duda genera una profunda crisis política y de valores que afecta la imagen externa del Ecuador, lo que tiene un efecto directo sobre riesgo país, valor que alcanzó una cifra récor histórica en el 2020, superando los 6000 puntos. 


\section{Microambiente.}

El estudio del microambiente permite conocer las fuerzas que actúan directamente sobre la empresa Campo Fino y sus similares, sus fortalezas y debilidades, así como evaluar su desempeño actual, recursos y fuerzas impulsoras.

El microambiente comprende el análisis de las relaciones del sector lácteo, los proveedores, los intermediarios, los sustitutos, el mercado de clientes potenciales y reales, y los públicos.

Para el estudio del microentorno se utiliza el modelo del entorno empresarial, el cual permite identificar los actores externos e internos que influyen de alguna forma en el sector financiero.

\section{La empresa.}

Campo fino empieza sus actividades en noviembre del 1999 en está catalogada como una MPYMES mediana, sus líneas de producción son: elaboración del: yogurt, queso y leche fresca pasteurizada.

\section{La competencia.}

Existen una gran cantidad productores artesanales de yogurt y queso fundamentalmente que en muchos casos realizan venta directa o en su defecto sus líneas de intermediación no superan a dos actores, también existen alrededor de 32 empresas de procesamiento de lácteos con cobertura regional y nacional cuyo sistema de producción se encuentra formalizado con cobertura en los principales centros de e expendio y comercialización (Supermercados, tiendas de abastos, mini mercados) sin embargo las más representativas son:

- Alpina productos alimenticios "ALPIEACUADOR S. A"

- Industrial láctea TONI

- Alimentos Chontalac CIA. LTDA.

- FLORALP S.A.

- Lácteos Santillán.

- Lácteos San Antonio C.A.

- PROLACHIV S.A

- Pasteurizadora el Ranchito CIA LTDA.

- REYBANPAC Rey Banano del Pacífico.

- LA FINCA CIA. LTDA.

- REY LACTEOS. 


\section{Los proveedores.}

Los principales proveedores de nuestra empresa son los pequeños productores de leche del cantón y provincia de Cotopaxi, pagamos el precio oficial por litro de leche, y adicionalmente les proveemos de ciertos servicios que generan fidelidad en cuanto a mantener la distribución de su producto hacia nuestra empresa.

\section{Intermediarios.}

Son las principales tiendas de abastos y supermercados locales que venden al menudeo nuestros productos.

\section{Mercado.}

Nuestro mercado es local es decir que nuestras a cadenas de distribución se encuentran ubicadas al interior de la provincia de Cotopaxi sin embargo pretendemos tener un alcance regional, para lo cual vamos a realizar inversiones en tecnología y en la búsqueda de proceso administrabas financiero más eficiente que permitan alcanzar un crecimiento sostenido.

\section{Diagnóstico estratégico.}

Previa a la implementación del diagnóstico estratégico se realizó encuestas al personal administrativo y operativo con el fin de establecer los principales problemas por las que atraviesa la empresa y partir de esta información poder orientar la aplicación del análisis FODA que permite estudiar los factores Interno (Fortalezas, debilidades) y externos que rodean a la organización, esto sin duda genera un valor agregado a los resultados ya es un proceso que se construye con la participación de todos los actores que intervienen en la cadena productiva de la empresa.

Para la aplicación de esta fase se planteó la realización de 3 encuentros (reuniones de trabajo): la primera es para la aplicación de las encuestas al personal operativo y administrativo, y la realización de la entrevista a los ejecutivos de la empresa, la segunda es de recolección de datos, la tercera es de validación de los resultados encontrados e interpretados por el equipo investigador. A continuación, se presenta los resultados de las encuestas y la matriz FODA con los resultados encontrados:

\section{Resultados de la encuesta.}

Tabla 1. Resultados de las encuestas aplicada a los trabajadores de la empresa Campo Fino 
1. ¿Existe una Comunicación eficiente que genere empoderamiento y alineamiento estratégico de los colaboradores con respecto a la misión, visión, valores y objetivos?

2.¿ La organización ha generado un manual de funciones defina con claridad las responsabilidades de todos quienes son parte de la empresa?

3.¿Existe un plan de Marketing que defina las estrategias y proceso que permitan que sus productos lleguen a otros segmentos del mercado?

4. ¿La capacidad de producción actual es suficiente para generar una eficiente sostenibilidad económica y financiera de la empresa?

5. ¿Se ha generado un incremento en los niveles de producción?

6. ¿Cuál es su opinión con respecto a la calidad de la materia prima y su influencia en la estandarización de la producción?

7. ¿Se realiza controles de calidad al producto final permanentemente?

8. ¿La calidad de los productos finales, ha permitido posicionar la marca en el mercado?
En un $17 \%$ que corresponde al área ejecutiva y administrativa, señalan que esta empoderada, el $83 \%$ manifiesta que algo conoce pero que no entiende para cual es el propósito de tener misión, visión.

6\% que corresponde al área ejecutiva y administrativa señala que existe cierto reglamentos que norman su funciones y responsabilidades, la diferencia que es del área operativa manifiesta que no existe un manual de funciones que norme su trabajo y actividades.

El $100 \%$ de los entrevistados manifestaron que no existen unas estrategias orientadas a captar nuevos clientes que el mercado con el que hoy cuenta la empresa es el que se ha logrado por gusto y fidelidad de los clientes hacia los productos.

El 22\% de los encuestados manifiestan que la empresa ha tenido recursos para poder solventar su funcionamiento, mientras que el $78 \%$ manifiesta que la empresa ha tenido dificultades económicas.

El 100\% manifiesta que sí se ha incrementado los niveles de producción de la empresa pero el ritmo es lento pese a los años que la organización está en el mercado El

El $100 \%$ manifiesta que existe dificultades con respecto a este tema debido a la diversidad de proveedores de leche y a su diferente categorización técnica en cuanto a sus sistemas productivos, por lo que se requiere generar proceso de fidelización a través de programas que ayuden a nuestros proveedores de leche, de tal manera que mejores la calidad el producto.

El control de calidad es permanente y es considerada una norma habitual dentro del proceso productivo.

El $100 \%$ considera que los productos son de buena calidad sin embargo la marca tiene un débil posicionamiento en el mercado esto se refleja el lento crecimiento de la empresa 
9.¿Existe el compromiso de todos quienes integran la empresa Campo Fino de contribuir con su esfuerzo y trabajo a posicionar nuevos mercados y de esta forma contribuir al fortalecimiento y sostenibilidad de la empresa?
Con respecto a este tema el personal manifestó el compromiso permanente por contribuir de forma positiva al crecimiento de la empresa

Fuente: Elaboración propia.

\section{Matriz FODA.}

Tabla 2. FODA de la empresa

\section{Factores internos}

\section{Fortalezas}

- Precios competitivos de sus productos en el mercado.

- Canales de distribución estables.

- Manejo de normas iso

- Acceso sostenido a materia prima de.

- Disponen de un plan de producción.

\section{Debilidades}

- Ambiente laboral inestable

- No existe un plan de incentivos a sus colaboradores.

- Deficiente posicionamiento de la marca en el mercado

- Estructura organizacional deficiente que afecta a los procesos de producción y empoderamiento del personal.

- Falta de capital para inversión

\section{Factores externos}

\section{Oportunidades}

- Mercado en crecimiento permanente.

- Disponibilidad de medios electrónicos que facilitan la difusión y venta en línea de sus productos.

- Acceso a sistemas de capacitación en línea a bajo costo.

- Existencias de políticas públicas que facilitan la presencia de sus productos en las grandes cadenas de supermercados nacionales.

\section{Amenazas}

- Competencia con un fuerte posicionamiento en el mercado.

- Inestabilidad política y económica del país.

- Inexistencia acceso a líneas de crédito específicas para productores.

- Mercado informal que genera desleal competencia.

- Falta de control de política pública que garantice acceso a materia prima de calidad.

Elaborado por: Los autores. 


\section{Diseño de estrategias.}

Tabla 3. FODA estratégico

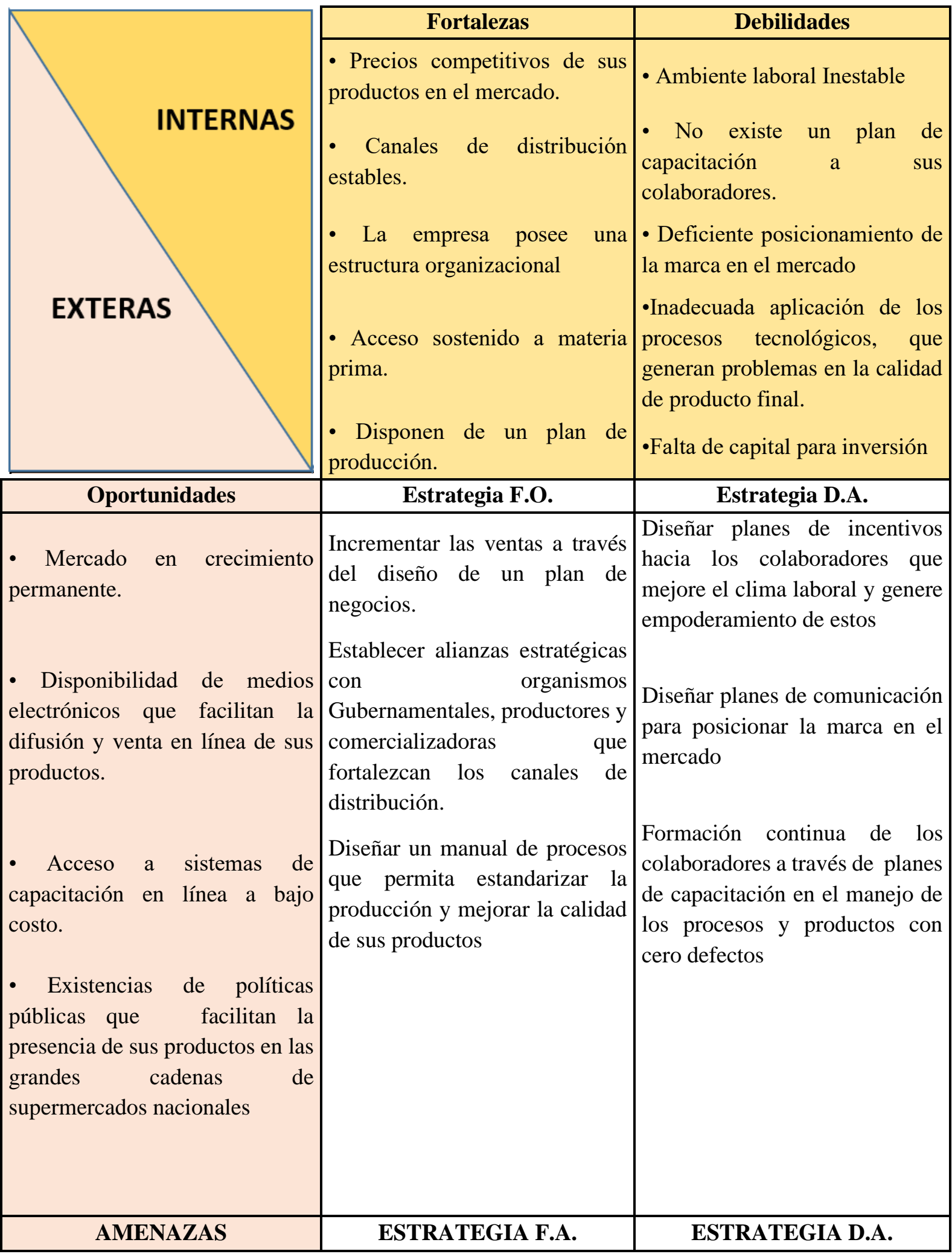


- Competencia con un fuerte posicionamiento en el mercado.

- Inestabilidad política y económica del país.

- Inexistente acceso a líneas de crédito específicas para productores.

- Mercado informal que genera desleal competencia.

- Falta de control de política pública que garantice acceso a materia prima de calidad.
Gestionar ante los organismos Implementar un plan de competentes la generación de comunicación que permita incentivos a través de política fortalecer la marca en el pública que genere una ventaja mercado competitiva a los pequeños y medianos productores.

Fuente: Elaboración propia.

\section{Fase filosófica.}

\section{Misión.}

Elaborar productos alimenticios de alta calidad garantizando la salud de nuestros consumidores, que generen confianza y fidelidad en nuestros clientes y posicionamiento de nuestra marca en el mercado de lácteos del país.

\section{Visión.}

Ser una empresa líder, consolidada y con alta participación en el mercado nacional de lácteos, sustentada sobre proceso técnico y organizacional que garantice la sostenibilidad económica financiera de la empresa.

\section{Valores Institucionales.}

\section{Trasparencia.}

La empresa campo fino y sus colaboradores, se compromete a construir acciones y procesos basados en la trasparencia y cumplimiento de las normas legales, morales y éticas.

\section{Compromiso.}


La empresa se compromete a cumplir con todas las normas técnicas que garanticen productos de alta calidad y sin riesgo para sus consumidores, a cumplir con las normas legales del país con respecto a la relación laboral con sus colaboradores y a cumplir con las responsabilidades asumidas con sus clientes y proveedores.

\section{Responsabilidad Social.}

Responsabilidad social con sus proveedores y pagar el precio justo por la materia prima para la elaboración de sus productos.

\section{Trabajo en equipo.}

Respetar, valorar la opinión de todos quienes son parte de la empresa y en un ambiente de fraternidad construir proceso consensuados que garanticen el empoderamiento de todos quienes son parte de la empresa campo fino.

\section{Liderazgo participativo.}

Que garantice una comunicación fluida y eficiente de doble vía, de tal forma que todas las acciones tengan la orientación adecuada para el cumplimiento óptimo de las actividades.

\section{Objetivos estratégicos.}

- OE1. Incrementar la sostenibilidad económica financiera de la empresa, a través del diseño e implementación de planes que mejoren su rentabilidad

- OE2. Incrementar la satisfacción de nuestros clientes con respecto a los productos y servicios que la empresa oferta, a través de mecanismos que permitan valorar el nivel de servicio que la institución presta.

- OE3. Optimizar la gestión institucional, a través del diseño, técnicas y metodologías que mejoren los tiempos de respuesta de la empresa.

- OE4. Incrementar el nivel de eficiencia y eficacia del talento humano a través de programas de capacitación que mejoren el rendimiento y generen empoderamiento.

\section{Fase táctica y operativa.}

Tabla 4. Fase táctica y operativa

\begin{tabular}{|c|c|c|c|}
\hline Objetivos estratégicos & Metas & Indicador & Plan acción \\
\hline Incrementar & Incremento & & Elaboración de un Plan de \\
\hline sostenibilidad & $\operatorname{del} 3$ & & para determinar mediante un \\
\hline $\begin{array}{l}\text { económica financiera } \\
\text { de la empresa, a través }\end{array}$ & $\begin{array}{l}\text { ROE sobre } \\
\text { la línea base. }\end{array}$ & & $\begin{array}{l}\text { estudio de mercado el incremento } \\
\text { de ventas. }\end{array}$ \\
\hline
\end{tabular}


ISSN: 2602-8506

Vol. 4, N³, p. 181-203, julio-septiembre, 2020

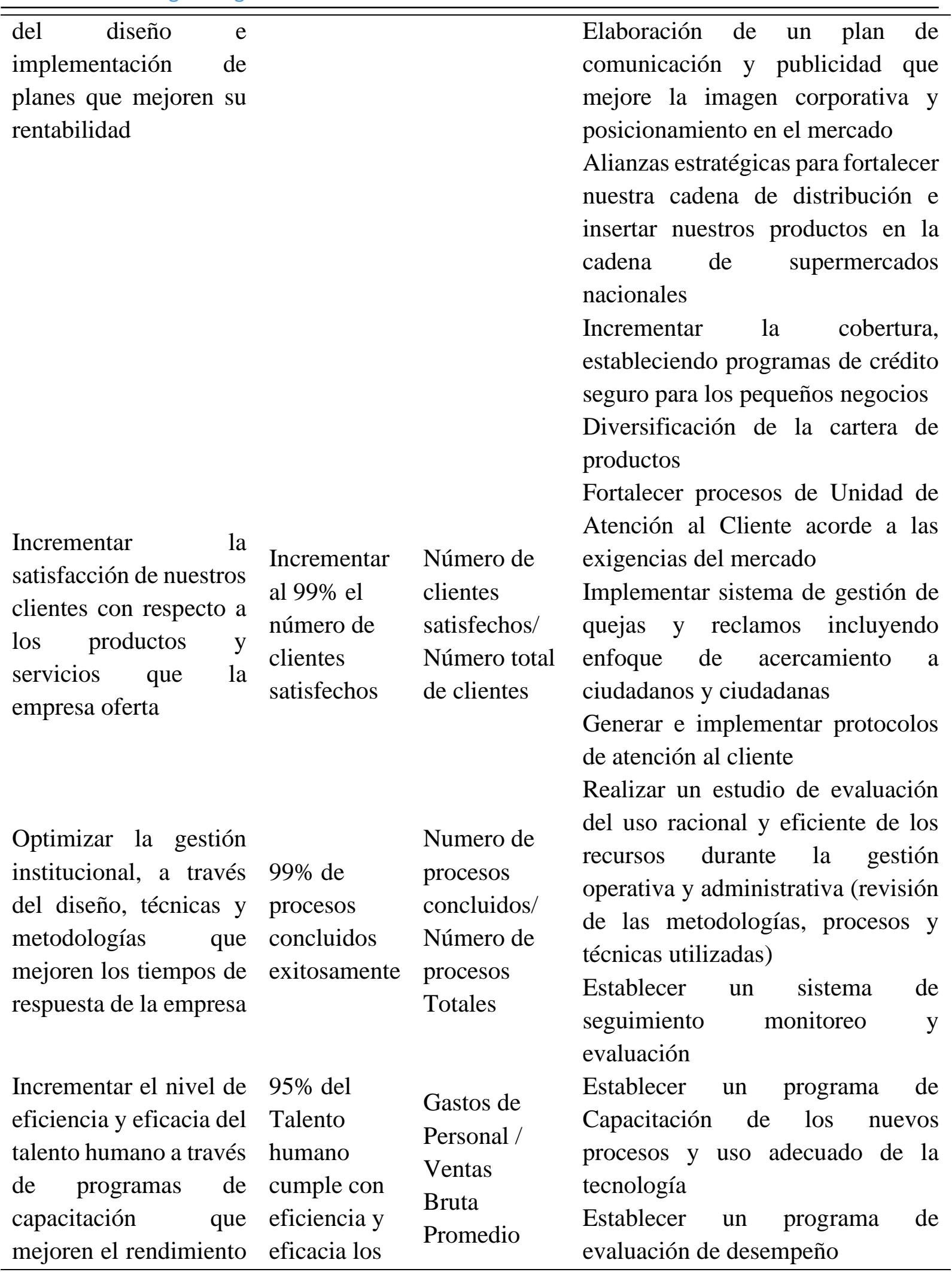




\begin{tabular}{lll}
\hline y generen & roles & Optimizar el talento humano, a fin \\
empoderamiento. & asignados. & de aprovechar las habilidades y \\
& conocimientos \\
& Exigir el uso adecuado de los \\
& bienes, suministros \\
& Establecer un sistema de trabajo por \\
& objetivos
\end{tabular}

Fuente: Elaboración propia.

\section{Conclusiones.}

- Al analizar la gestión de la empresa se determinó que muchos de los procesos que se llevan en su pese a tener definido ciertos procedimientos solo se aplican en la generalidad de los casos por el personal administrativo, mientras que el operativo se maneja más por la experiencia que cada uno de ellos tienen en la elaboración de productos, esto sin duda genera dificultadas al querer estandarizar la producción y mejorar la calidad de estos.

- La actitud y compromiso del personal es buena pero lamentablemente no existe canales adecuados y eficientes de comunicación entre la administración y la parte operativa que permita integrar el trabajo en equipos eficientes y que este valor se vea reflejado en el incremento de la productividad, al contrario, muchas veces esta actitud no correspondida genera frustración lo que afecta el clima laboral y las relaciones armónicas que deben tener todos quienes trabajan en la empresa.

- La empresa en el trascurso del tiempo desde su creación ha logrado construir cierto mercado, pero lamentablemente no ha crecido en la dimensión que los propietarios buscan debida a que no se han aplicado procesos técnicos en la gestión que permita posicionar la marca en un mercado cada vez más competitivo.

- No existe un sistema que permita medir con eficiencia y efectividad el desempeño de cada uno de componentes de la empresa esto sin duda tiene un efecto fundamental en el cumplimiento de las metas y objetivos lo que da como resultado un crecimiento extremadamente lento con respecto a otras empresas del mismo segmento.

- Otro aspecto para destacar es que en la empresa no se han diseñado planes de capacitación e incentivos lo que afecta el desempeño de los colaboradores, sumado a esto que no existe un modelo que permita medir el desempeño laboral de cada uno de los que conforman la empresa campo fino. 


\section{Referencias bibliográficas.}

Armijo, M. (2014). Planificación estratégica e indicadores de desempeño del sector público. Recuperado de: https://www.cepal.org/ilpes/publicaciones/xml/8/44008/SM_69_MA.pdf.

Mora, L 2019. Plan estratégico para mejorar la eficiencia de los procesos administrativos financieros en la empresa de lácteos "CAMPO FINO", de la ciudad de Salcedo durante el periodo 2018 - 2023, Tesis de pregrado Escuela Superior Politecnica de chimborazo, recuperado de http://dspace.espoch.edu.ec/bitstream/123456789/11303/1/22T0503.pdf

Blandez, M. (2014). Proceso administrativo. Recuperado de: https://books.google.com.ec/books?hl=es\&lr=\&id=TYHDCwAAQBAJ\&oi=fnd\&p $\mathrm{g}=\mathrm{PT} 6 \& \mathrm{dq}=$ todo+sobre+proceso+administrativo+financiero+\&ots=pnsdlGXFJT\&s ig $=$ NodS1a00dVHmpgc0vbwqE_fPKRs\&redir_esc $=y \# v=$ onepage\&q=todo $\% 20$ sobr e\%20proceso\%20administrativo\%20financiero\& $\mathrm{f}=$ false

Chiavenato, I. (2007). Introducción a la teoría general de la admiistración. Recuperado de: https://naghelsy.files.wordpress.com/2016/02/introduccic3b3n-a-la-teorc3adageneral-de-la-administracic3b3n-7ma-edicic3b3n-idalberto-chiavenato.pdf

Chiavenato, I. (2011). Planeación estratégica. Fundamentos y aplicaciones. $2^{\mathrm{a}}$. ed. México: McGraw-Hill.

Ekos. (05 de Abril de 2017). Productos lácteos: menor cosumo. Ekos. Recuperado de:http://www.ekosnegocios.com/negocios/verArticuloContenido.aspx?idArt=8909

Graffe, G. (2006). La planificación, modalidades y el uso de modelos. Recuperado de:https://cursos.campusvirtualsp.org/repository/coursefilearea/file.php/61/bibli/m3planificacion_modalidades_y_el_uso_de_modelos.pdf

Guerrero, E. (2016). Técnicas e instrumentos. Recuperado de: http://www.medic.ula.ve/tsues/TECNICAS_E_INSTRUMENTOS.pdf

INEC. (2017). Encuesta de superficie y producción agropecuaria continua. Recuperado de: http://www.ecuadorencifras.gob.ec/documentos/webinec/Estadisticas_agropecuarias/espac/espac_2017/Informe_Ejecutivo_ESPAC_201 7.pdf

Morales, A. \& Morales, J. (2014). Planeación financiera. Recuperado de: https://editorialpatria.com.mx/pdffiles/9786074382167.pdf

Muilema, G. (2011). Proyecto de factibilidad para la creación de una empresa láctea en la parroquia Toacaso, cantón, Latacunga, Provincia de Cotopaxi. (Tesis de 
ISSN: 2602-8506

www.visionariodigital.org

Vol. 4, $\mathrm{N}^{\circ} 3$, p. 181-203, julio-septiembre, 2020

pregrado,Universidad Técnica de Cotopaxi). Recuperado de: http://repositorio.utc.edu.ec/bitstream/27000/1266/1/T-UTC-1308.pdf

Ortega, A. (2008). Planeación financiera estratégica. México: McGraw-Hill.

Rosero, D. (2013). Plan de mejoramiento para la implementacion de planeacion estratégica en la empresa quesera los guaduales. (Tesis de pregrado,Universidad San Buenaventura). Recuperado de: https://bibliotecadigital.usb.edu.co/bitstream/10819/2056/1/Plan\%20de\%20Mejora miento_Quesera\%201os\%20Guadales_Rosero2013.pdf

Sampieri, R. (2014). Metodología de la investigación. Recuperado de: https://periodicooficial.jalisco.gob.mx/sites/periodicooficial.jalisco.gob.mx/files/met odologia_de_la_investigacion_-_roberto_hernandez_sampieri.pdf

Sierra, H.\& Bernal, C. (2008). Proceso Administrativo para las organizaciones del siglo XXI. México: Pearson. 
PARA CITAR EL ARTÍCULO INDEXADO.

Edison Ruperto Carrillo Parra, E. R. C. P., Rodríguez Solarte, Ángel E., \& Arellano Díaz, M. A. (2020). La planificación estratégica como herramienta de desarrollo de las empresas agropecuarias en el Ecuador. Visionario Digital, 4(3), 181-203. https://doi.org/10.33262/visionariodigital.v4i3.1361

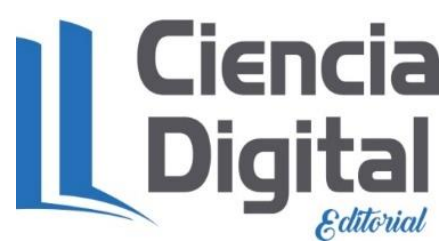

El artículo que se publica es de exclusiva responsabilidad de los autores y no necesariamente reflejan el pensamiento de la Revista Visionario Digital.

El artículo queda en propiedad de la revista y, por tanto, su publicación parcial y/o total en otro medio tiene que ser autorizado por el director de la Revista Visionario Digital.
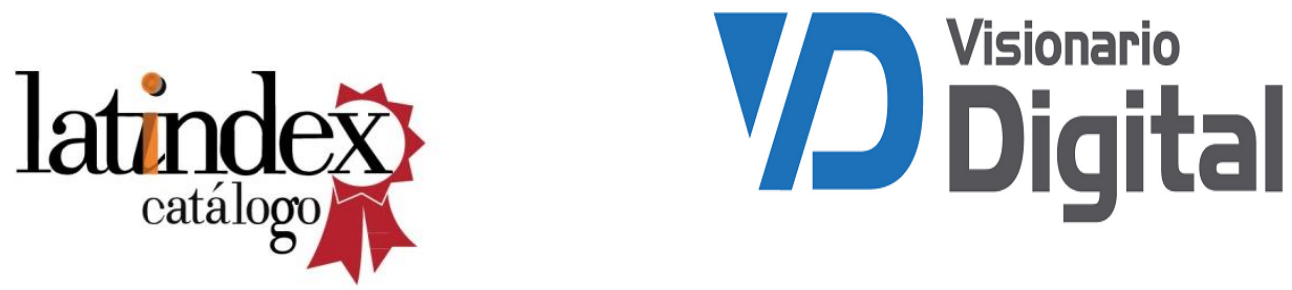